\title{
Towards a more universal life detection strategy
}

White Paper submitted to the Planetary Science and Astrobiology Decadal Survey 2023-2032
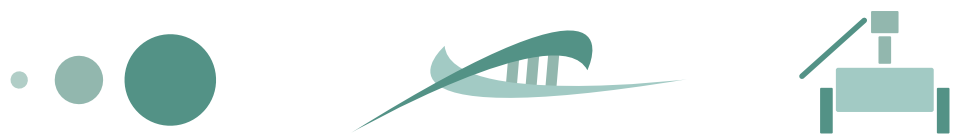

\section{Lead authors:}

Luoth (Lu) Chou

NASA Goddard Space Flight Center, Greenbelt, MD, USA

Georgetown University, Washington, DC, USA

luoth.chou@nasa.gov | +1-301-286-5220

Natalie Grefenstette

Santa Fe Institute, Santa Fe, NM, USA

Blue Marble Space Institute of Science, Seattle, WA, USA natalie.grefenstette@santafe.edu | +1-505-946-2722

\section{Co-authors:}

Sarah S. Johnson (Georgetown University)

Heather Graham (NASA Goddard Space Flight Center)

Paul Mahaffy (NASA Goddard Space Flight Center)

Christopher Kempes (Santa Fe Institute)

Jamie E. Elsila (NASA Goddard Space Flight Center)

Eric Libby (Umeå University)

Andrew Ellington (University of Texas, Austin)

Eric Anslyn (University of Texas, Austin)

Tori Hoehler (NASA Ames Research Center)

Peter Girguis (Harvard University)

Leroy Cronin (University of Glasgow)

William Brinkerhoff (NASA Goddard Space Flight Center)

Barbara Sherwood Lollar (University of Toronto) 
Co-signers:

\begin{tabular}{|c|c|}
\hline Joshua Anadu ${ }^{1}$ & Chetanath Neupane ${ }^{26}$ \\
\hline Dale T. Andersen ${ }^{2}$ & Marc Neveu ${ }^{11,27}$ \\
\hline Stuart Bartlett ${ }^{3}$ & Julie Nekola Novakova ${ }^{28,29}$ \\
\hline David A. Baum ${ }^{4}$ & Siddharth Pandey ${ }^{30,5}$ \\
\hline 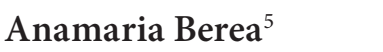 & Niki Parenteau $^{7}$ \\
\hline Julie Bevilacqua $^{6}$ & Ivan G. Paulino-Lima ${ }^{5}$ \\
\hline Jennifer G. Blank ${ }^{5,7}$ & Alexandra Pontefract ${ }^{6}$ \\
\hline Schuyler Borges ${ }^{8}$ & Chad I. Pozarycki ${ }^{11,12}$ \\
\hline Tristan Caro ${ }^{9}$ & Laura Ratliff 6 \\
\hline Pamela G. Conrad ${ }^{10}$ & Aaron B. Regberg ${ }^{31}$ \\
\hline Victoria Da Poian ${ }^{11,12}$ & Andro C. Rios ${ }^{5}$ \\
\hline Ryan M. Danell ${ }^{11,13}$ & Edgard G. Rivera-Valentín ${ }^{32,33}$ \\
\hline Jason P. Dworkin ${ }^{11}$ & Anaïs Roussel ${ }^{6}$ \\
\hline Jennifer Eigenbrode ${ }^{11}$ & Mary Sabuda ${ }^{34}$ \\
\hline R. Craig Everroad ${ }^{7}$ & Micah J. Schaible 35 \\
\hline G. Matthew Fricke ${ }^{14}$ & Lana Sinapayen ${ }^{36}$ \\
\hline Tyler H. Garvin ${ }^{15}$ & Hillary H. Smith ${ }^{17}$ \\
\hline Stephanie A. Getty ${ }^{11}$ & Evan L. Sneed ${ }^{17}$ \\
\hline Daniel P. Glavin ${ }^{11}$ & Sanjoy Som ${ }^{5}$ \\
\hline Roland Hatzenpichler ${ }^{16}$ & Andrew Steele ${ }^{10}$ \\
\hline Christopher House ${ }^{17}$ & Marshall J. Styczinski ${ }^{37}$ \\
\hline Alta Howells ${ }^{18}$ & Wesley D. Swingley ${ }^{38}$ \\
\hline Andrew Hyde ${ }^{17}$ & George K. Tan ${ }^{35}$ \\
\hline Tony Z. Jia ${ }^{19,5}$ & Bethany P. Theiling ${ }^{11}$ \\
\hline Daniel S. Jones ${ }^{20,21}$ & Gareth Trubl ${ }^{39}$ \\
\hline Desmond A. Kaplan ${ }^{11,22}$ & Lena Vincent ${ }^{4}$ \\
\hline Fabien Kenig ${ }^{23}$ & Nicole Y. Wagner ${ }^{6}$ \\
\hline Judy Malas ${ }^{23}$ & Margaret M. Weng 6 \\
\hline Michael J. Malaska $a^{24,3}$ & Michael L. Wong ${ }^{37}$ \\
\hline Maëva Millan ${ }^{11,6}$ & Yuk Yung ${ }^{3}$ \\
\hline
\end{tabular}

Karan Molaverdikhani ${ }^{25}$

Affiliations:

${ }^{1}$ Oklahoma State University, ${ }^{2}$ SETI Institute, ${ }^{3}$ California Institute of Technology, ${ }^{4}$ University of Wisconsin-Madison, ${ }^{5}$ Blue Marble Space Institute of Science, ${ }^{6}$ Georgetown University, ${ }^{7}$ NASA Ames Research Center, ${ }^{8}$ Northern Arizona University, ${ }^{9}$ University of Colorado, Boulder, ${ }^{10}$ Carnegie Institution of Science, ${ }^{11}$ NASA Goddard Space Flight Center, ${ }^{12}$ Center for Research and Exploration in Space Science \& Technology (CRESST), ${ }^{13}$ Danell Consulting, Inc., ${ }^{14}$ University of New Mexico, ${ }^{15}$ Harvard University, ${ }^{16}$ Montana State University, ${ }^{17}$ Pennsylvania State University, ${ }^{18}$ Arizona State University, ${ }^{19}$ Earth-Life Science Institute (ELSI), ${ }^{20} \mathrm{New}$ Mexico Tech, ${ }^{21}$ National Cave and Karst Research Institute, ${ }^{22}$ KapScience LLC, ${ }^{23}$ University of Illinois at Chicago, ${ }^{24}$ Jet Propulsion Laboratory, ${ }^{25} \mathrm{Max}$ Planck Institute for Astronomy, ${ }^{26}$ Center for Astrobiology Research, Nepal, ${ }^{27}$ University of Maryland at College Park, ${ }^{28}$ Charles University, ${ }^{29}$ European Astrobiology Institute, ${ }^{30}$ Amity University India, ${ }^{31}$ NASA Johnson Space Center, ${ }^{32}$ Lunar and Planetary Institute, ${ }^{33}$ Universities Space Research Association (USRA), ${ }^{34}$ University of MinnesotaTwin Cities, ${ }^{35}$ Georgia Institute of Technology, ${ }^{36}$ Sony Computer Science Laboratories, ${ }^{37}$ University of Washington, ${ }^{38}$ Northern Illinois University, ${ }^{39}$ Lawrence Livermore National Lab 


\section{Executive Summary}

The most daunting challenge in the search for life elsewhere may be contending with truly alien life. Much of the astrobiology research in the past decade has driven mission science to search for well-established, recognizable biosignatures based on Terran life. However, the probability that life in the universe would share a biochemical ancestry with life on Earth quickly diminishes the further away from Earth we explore. Thus, it is imperative that we build foundational knowledge for life detection strategies that target universal biosignatures.

This report advocates that NASA invest in the development and implementation of a more inclusive framework for universal biosignatures detection. We recommend that such a framework be used to guide mission and instrument selection. Specifically we urge: (1) strong and sustained support of R\&A to develop universal theories to recognize life, along with an increase in the breadth of interpretations of returned mission data; (2) support of a more diverse program of instrument developments and maturations; and (3) examination of a wider range of complementary in situ science measurements using robust statistical methods.

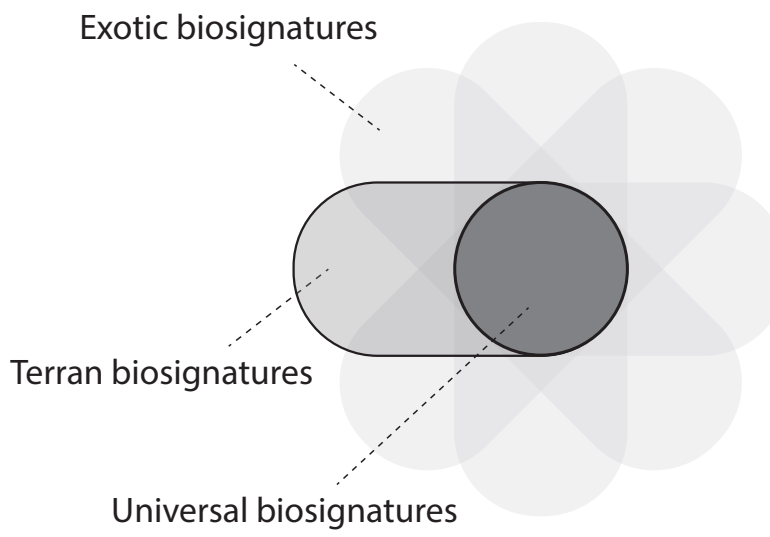

Figure 1: A conceptual representation of possible biosignatures that could exist in the universe. 'Terran biosignatures' applies specifically to biological features based on life on Earth and are not universal features of all life forms. 'Exotic biosignatures' refers to specific features displayed by potential life forms outside of Earth. 'Universal biosignatures' are features that are common to all possible life forms in the universe.

\section{A. Motivations: Why do we need to improve life detection?}

All possible forms of life, be it Terran or exotic, past or present, alive or dead, express potentially detectable signals termed biosignatures. Here, we depict a conceptual representation of biosignatures that could exist in the universe (Figure 1). This diverse spectrum of biosignatures contains commonalities that are universal features of all biology. In this paper, we define these features as "universal biosignatures" (also known as "agnostic biosignatures"). As one moves away from the center circle in Figure 1, the biosignatures become more specific to a particular evolutionary history, and thus, to the particular environmental context of a planetary body. It is important to note that while it is reasonable to assume that distinct origins of life will yield diverse biochemistries and macromolecules, how different exotic biosignatures will be from Terran biosignatures is formally an open question. Here, we operate under the assumption that life is diverse in the universe, but acknowledge that there is no existing science or theory that tells us how diverse biochemistries are across the universe. Terran-only biosignatures, as discussed here, are features that are specific to Terran biology (i.e., using DNA/RNA as informational polymers, diagnostic molecular fossils in the rock record, or even the presence of $\mathrm{O}_{2}$ in our atmosphere, to name a few) and epitomize our one example of biology. Most of our current life detection strategies stem from this single, limited example, in particular by leveraging what we know (Figure 2, in yellow) and what is detectable with our current technology (Figure 2, in blue). 


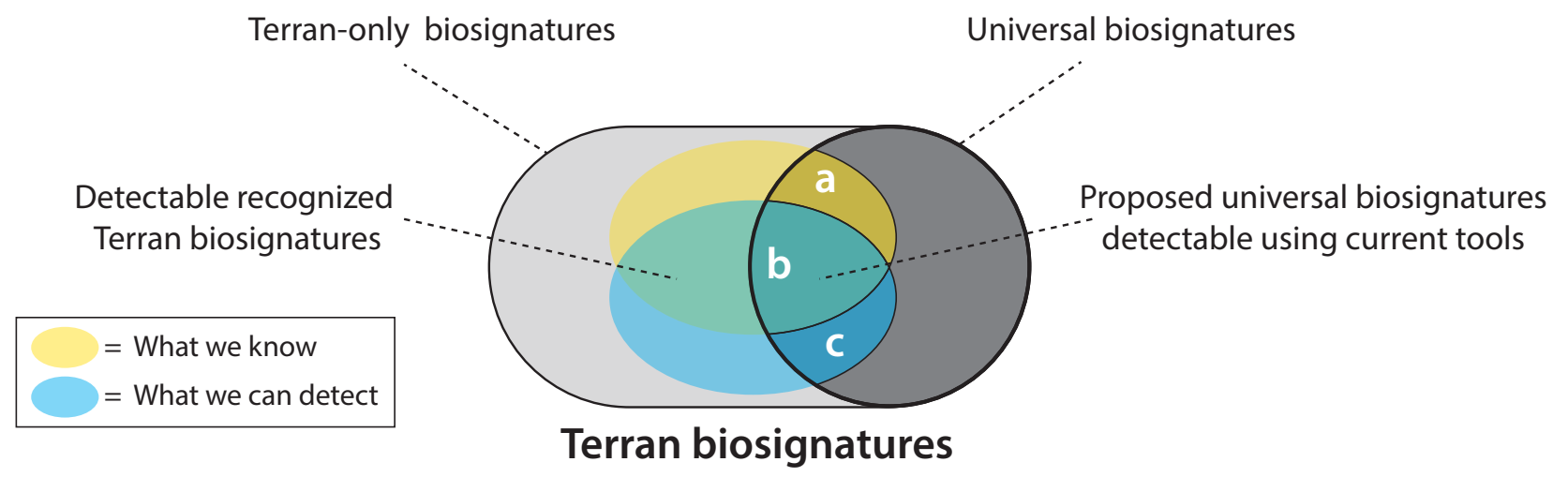

Figure 2: A conceptual representation of the state of Terran and universal biosignature knowledge and detection capabilities (based on Figure 1). We define different potential research focuses and implementation targets: (a) proposed theoretical universal biosignatures we can't currently detect, (b) proposed universal biosignatures we can detect using current tools, and (c) detectable but unrecognized universal biosignatures.

As a consequence of this narrowly focused exploration effort, we risk missing signs of life based on an unfamiliar biochemical system. Due to the cost and limited opportunities to look for life with space missions, it is vital that future life detection missions ensure they are casting the widest possible net by looking for signatures of life that are potentially universal to all possible life forms. This generalized strategy is a spending priority as it reduces the chance of a false-negative (contingent on our technological capabilities) and thus increases the chance of a successful science return. This is especially important in light of recent exciting discoveries about liquid environments on solar system worlds such as Mars (Orosei et al., 2018) and Europa (Jia et al., 2018), as well as organics on Titan (Niemann et al., 2010), Enceladus (Postberg et al., 2018), and Mars (Eigenbrode et al., 2018). Furthermore, recent advances in technologies such as innovative mobility solutions planned for Titan (NASA, 2019) and modern launch capabilities such as the Space Launch System (Klaus et al., 2013) widen the possible exploration targets and mission architectures to distant and unfamiliar worlds such as Europa (Hand et al., 2017) and Titan (NASA, 2019).

\section{B. Recommendations for generalizing life detection strategies}

In order to successfully generalize our approach to life detection, we need to (1) broaden our knowledge base by understanding what features of Terran life are likely universal (Figure 2, Segments a \& b), (2) expand our detection capabilities to match our state of knowledge of universal biosignatures (Figure 2, Segments $b$ \& $c$ ) and (3) implement current and future methods proposed to detect universal biosignatures that are based on developed technologies and new theory (Figure 2 , Segment $b$ ). Jointly, these steps increase our ability to detect life by steering exploration objectives from looking for features that are specific to life on Earth (Figure 1, Terran-only biosignatures), to looking for general features of life (Figure 1, Universal biosignatures) that, importantly, includes detecting Terran-like life.

\section{(1) Broadening our knowledge base}

To detect life beyond Earth, we first need to delineate what we are looking for. Even without a complete understanding of what defines a living system, life exhibits unique combinations of features not 
found in abiotic systems. As such, we need to identify and understand those features. There are two aspects of universal biosignatures that must be considered: is the proposed universal signature, or combination of signatures, robust (can it be used to distinguish life from non-life, discussed in Section C) and is it truly universal (can it be generalized to life beyond Earth).

Concerning the latter, it is important

Aspects of universal life detection strategies that need to be considered:

$\rightarrow \quad$ Finding and implementing detectable universal biosignatures by:

$\triangleright \quad$ Broadening our knowledge base

$\triangleright$ Expanding our detection capabilities

$\triangleright$ Implementing current proposed universal biosignatures

$\rightarrow$ Strengthening the robustness of life detection by:

Establishing a reference sample set

Using complementary approaches to measurements

Enhancing our contamination knowledge

to note that without at least another data point for life, any proposed universal signature remains an untestable hypothesis. That being said, we posit that insight into potential universal biosignatures can be harnessed from the one example of life that we have here on Earth, as outlined below. Knowledge concerning potential universal biosignatures can come from two types of approaches: bottom-up and top-down.

1. A bottom-up approach here refers to research being conducted on universal principles of biology, physical laws of biology, complexity and emergence of life (Kim et al., 2019; Walker, 2019; Kempes et al., 2019; Libby et al., 2019). It is important to support strong modeling, theoretical, and even experimental research in these areas in order to strengthen our understanding of what makes life different from abiotic systems at the most fundamental level. This kind of principles-first approach can then lead to hypotheses about which key differences between the biotic and abiotic world could be reflected on a physical level and thus might constitute detectable signatures of living systems (past or present). Whether these proposed universal patterns are robust biosignatures then needs to be verified (using existing data or the reference sample set described in Section C.1).

2. A top-down approach here refers to searching for distinguishable features of Terran life and abiotic chemistry by examining data from various types of biotic and abiotic samples in order to reveal key differences between data stemming from biology or not (Chan et al., 2019). This could be achieved through analyzing comprehensive data sets (Section C.1) or looking at existing data in new ways. This approach can lead to the discovery of robust biosignatures, some of which might be universal. Whether these proposed biosignatures are in fact generalizable to life beyond Earth then needs to be supported by strong theoretical evidence, in order to validate these as proposed universal biosignatures.

Knowledge gained from these two approaches can also yield insights into what measurable imprints are hypothetically possible in a physical and chemical space in the absence of life, and thus allow for a more compelling rejection of a false positive or false negative interpretation. Additionally, these approaches can help refine the measurement requirements necessary for the detection of probable universal biosignatures. Meaningful insights into what could constitute a useful universal biosignature lie at the interface between hypotheses obtained from theoretical studies and those extrapolated from the data we have access to. Thus it is vital to support close collaborations between these two key approaches. 
(2) Expanding our detection capabilities In parallelwith an increased understanding of universal biosignatures (Section B.1), improved detection capabilities are needed. This can be achieved by (1) taking advantage of existing high heritage instrumentation to perform broader science measurements that could encompass universal biosignatures, (2) supporting technology development that brings proof-of-concept studies and instrumentation closer to flight readiness, and (3) developing new measurement capabilities to match newly proposed universal biosignatures that revealed a blind spot in our current technological capabilities.

Employing existing high heritage technology is not only a cost-effective strategy, but also ensures higher success during future mission implementation. For example, conventional means to detect unequivocal Terran organic biosignatures (e.g., molecular fossils, or biogenic gases) utilize miniaturized mass spectrometers that have prevailed in space exploration since the 1970's (Biemann, 1979; Mahaffy et al., 2012). Because these instruments could also reveal intrinsic properties of molecules (such as fragmentation patterns or structures), they can be fruitful tools to help decipher potential universal biosignatures that may be represented in such data (Section C.3). However, a reconceptualization of the measurement requirements might be necessary in order to make the measurements more inclusive to potential universal biosignatures. These revised requirements can be informed by theoretical or proof-of-concept studies, as discussed previously. Further, these requirements can be implemented in current and future missions such as those under development for Mars and Titan (e.g., Li et al., 2017; Goesmann et al., 2017; NASA, 2019).

Other proposed methods to detect universal biosignatures have advanced in technological developments, but have not yet achieved flight-readiness status. One such technique derives from the hypothesis that biological entities express an overabundance of diverse surface chemical expressions (e.g., a vast number of receptors on the surface of cells) relative to abiological substances (e.g., a few crystal faces of minerals). Regardless of the biochemistry of the purported biological entity, such differences in surface chemical diversity could potentially be detected (Stoltenburg et al., 2007; Sun and Zu, 2015). One proposed means of detection involves using the proximity ligation assay method that utilizes the ability of short strands of oligonucleotides ${ }^{1}$ to bind to those surface expressions. Combined with compact and low-power sequencing devices (e.g., nanopore ${ }^{2}$ ), such technology can provide a remarkable view into the complexity of surfaces (Johnson et al., 2018). In addition, enantiomeric enrichment of certain chiral compounds such as polyols are suggested to be a detectable universal biosignature, but are currently "beyond the capabilities of spaceflight instrumentation" (Glavin et al., 2020). Finally, all living systems by necessity maintain themselves in chemical disequilibrium from the environment. Hence detecting an unexpected chemical disequilibrium could serve as a universal biosignature. This could be achieved by employing an inert, conductive electrode (e.g., graphite) in the environment, to measure redox state, to catalyze redox reactions, and to look for sustained disequilibria such as temporally invariable electrical currents (see Gartman et al., 2017). Supporting the maturation of these proof-of-concept methods could dramatically increase the measurement capabilities to detect universal biosignatures in missions flown to search for evidence of life beyond Earth.

\footnotetext{
${ }^{1}$ Oligonucleotide: short DNA or RNA molecule

${ }^{2}$ Nanopore: third-generation miniature sequencing device capable of sequencing single DNA or RNA molecules
} 
Expanding our detection capabilities also requires support for the development of new instrument concepts (National Research Council, 2011) that would fill the need for currently undetectable universal biosignatures. For example, there are several key areas where we are currently lacking in detection capability, including the detection of polyelectrolytes (e.g., polymers with a repeating charge in their backbone have been proposed as essential to life; Benner, 2017), or more generally the detection of live organisms through the search for dynamic biosignatures such as responses to environmental changes or metabolic activity (detection of redox and other energy exchange reactions that are inconsistent with abiotic processes).

Experiments to detect metabolic activity were famously attempted during the Viking mission, the first mission where the core aim was life detection. The results from these experiments were widely considered to be inconclusive (Biemann, 1979; Klein, 1979; Clark, 2018), but not without dissenting viewpoints (Levin et al., 2016). Similar missions with experiments based on Terran microbial metabolism knowledge have not been attempted since. The muddled outcomes revealed that better understanding of the signatures of metabolism was necessary, as well as better planning of in situ science experiments on other planets, especially considering the unknown geochemical variables that could impact the reliability of the signals.

The steps necessary to develop or implement any universal biosignature detection technologies for targeted missions need to be established as a result of concerted transdisciplinary efforts. This should include discussions among fields such as instrument development, systems engineering, and mission development; but also astrobiology, in which critical formalization of high-level science objectives and measurement objectives can be made. Thus, we urge NASA to support and foster the community in this endeavor to establish a stronger partnership between instrument scientists and astrobiologists, not only during the conceptualization, planning, or development stage, but also the operations stage.

\section{(3) Current implementable proposed universal biosignatures}

Recent efforts to look for general features of life have yielded potential actionable universal biosignatures of promise. Notably, recent work has proposed to make use of high heritage instruments such as mass spectrometers (MS) in order to look at the complexity of molecular structures within a sample (by proxy of fragmentation patterns observed in the spectra). This research is based on the hypothesis that only specific stepwise reactions would yield the kinds of complex molecules seen in Terran biology, and as such, molecules beyond a certain threshold of complexity are more likely to be a product of metabolic reaction networks than a product of their abiotic environment (Marshall et al., 2017). Additionally, it has been proposed to make use of our knowledge of the relative abundances of compounds in samples analyzed, based on the hypothesis that living systems and abiotic systems will produce different distributions of compounds (Dorn et al., 2011), which can be obtained via MS. Another proposed universal biosignature that makes use of MS is the detection (and identification) of heteropolymers ${ }^{3}$. Heteropolymers hold a central role in Terran biology due to their ability to expand the functional chemical space, and their ability to store information in their sequences (information that can be accessed by the biological system, copied, propagated and mutated, allowing for evolution) (Hoehler et al., 2020). Thus, the detection of such polymers beyond Earth, regardless of the specific chemistry, has been argued as a potentially detectable universal biosignature (NASEM, 2018, Chou et al., 2020). Searches for these proposed universal biosignatures are implementable in current and future missions.

\footnotetext{
${ }^{3}$ Heteropolymer: a polymer consisting of two or more monomers
} 


\section{Recommendations for enhancing robustness of life detection}

Our goals are to make any life detection research or mission more inclusive to different forms of life, as well as making it more robust in order to strengthen our confidence in the event of life detection. To achieve these goals, we strongly recommend that NASA establish rigorous guidelines for instrument selection criteria and science measurement requirements that are informed by the following:

(1) A comprehensive reference sample set that contains materials spanning the spectrum from inorganic substances that are not generally associated with life (abiotic) to unambiguously biological material (biotic), and materials between them such as abiotic organic samples and degraded biological material (Conrad and Nealson, 2001). Selection of biotic and abiotic materials with different preservation potential should also be incorporated, consistent with the recommendations of the National Academies of Sciences "Astrobiology Science Strategy for the Search for Life in the Universe" (NASEM, 2018). This sample set serves as a "testbed" for interrogating potential universal biosignatures proposed through theoretical work, as well as for extrapolating potential universal biosignatures by extracting distinguishable features between biotic and abiotic samples (Section B.1). This sample set should be collectively studied across wide ranges of disciplines where measurements on multiple instrument platforms are obtained, and the data generated from such efforts should be sharable and accessible across institutions. These data should help continuously refine instrument selection criteria and possibly even inform mission selection criteria in order to maximize the chances of life detection beyond Earth. Finally, having an expansive and comprehensive dataset should also aid in the development of science autonomy applications (i.e., machine learning and artificial intelligence) that could be implemented in space, especially if critical autonomous decisionmaking is needed to enhance science return (Theiling et al., 2020).

(2) A checks-and-balances approach between scientific measurements that increases the definitiveness of potential biosignatures and decreases the likelihood of false positive or false negative interpretations. This recommendation stems from the argument that definitive life detection requires multiple lines of observations and that no single measurement is adequate to fully confirm biogenicity, in line with standard practices in other scientific disciplines. Therefore, we recommend that instruments be selected for life detection missions as suites or collective units rather than as stand-alone tools designed for specific and narrow measurements. Strong evidence is supported by multiple lines of measurements made on samples across scales (i.e., molecular, microscopic, or macroscopic) and time, within an integrated environmental, geological, and chronostratigraphical context (Steele et al., 2016). Any interpretation of biogenicity needs to be considered in the context of complementary background information (chemical, isotopic, mineralogical, physical) and thus payloads need to be capable of characterizing the framing environment so that the novelty of an observation can be confirmed (NASEM, 2018). We recommend using a Bayesian approach to help identify which suites of complementary methods should be used to impart higher confidence in the evidence perceived, thereby quantifying the likelihood that life was detected (NASEM, 2018). This approach could also help assign probabilities to more convoluted signals from ambiguous cases such as old life, degraded life, products of life (e.g. viruses), early life, and gray cases just preceding the emergence of life. 
(3) An increase in knowledge on potential contaminating signals not native to the sample being measured. These signals could stem from a myriad of sources, such as events (e.g., pre-flight handling, exposure to contaminants during flight or landing, etc), pathways (e.g., processes within the instrument that can lead to accumulation of certain compounds over time, space conditions leading to significant changes to the instrument, deterioration of instrument detection capabilities) or materials (e.g., parts within the instrument, including chromatography columns, derivatizing agents, etc, that can appear in the measurement). Some practices are often discussed in the context of possible (Terran) biological contamination as it pertains to space exploration (notably Planetary Protection practices, Rummel, 2019), however standard control measures should be put in place to address physical and chemical contamination, as discussed in Dworkin et al. (2018). This includes cataloging all possible contamination sources (chemical, physical, or biological) that could "cast a shadow" on the definitiveness of a signal. Additionally, universal biosignature methods could be applied to protocols for tracing contaminations on spacecraft before flight. Not only would these methods help capture signals that could convolute measurements, but they can also help account for contamination from potentially unrecognized Terran life or byproducts of life. These recommendations require a community effort to address the limitations and challenges associated with modern pre-flight contamination control and can be applied to any mission.

\section{Conclusion:}

As we broaden our exploration endeavors further into the solar system, the likelihood of encountering familiar life diminishes. Therefore, it is vital to expand the scope of life detection strategies to include universal biosignatures. We recommend focusing on research into universal features of life that can inform measurement requirements and guide data analysis and instrument selection, while accelerating the development of life detection technologies suitable for a more comprehensive search strategy. Strong and sustained support of R\&A and technology development in the area of universal biosignatures and life detection is essential to achieving these goals. A universal approach to life detection will significantly diminish the potential for false negative results that could arise from constraining our search to 'life as we know it', and thereby would enhance NASA's efforts to seek for evidence of life beyond Earth.

\section{References:}

Benner, S. A. Astrobiology 17, 840-851 (2017). Biemann, K. L. Mol. Evol. 14, 65-70 (1979). Chan, M. A. et al. Astrobiology 19, 1075-1102 (2019). Conrad, P. \& Nealson, K. Astrobiology 1 (1), 15-24 (2001). Chou, L. et al. LPSC 51, 2706 (2020). Clark, B.C. Handbook of Astrobiology, (2018). Dorn, E. D. et al. J. Mol. Evol. 72, 283-295 (2011). Dworkin, J. P. et al. Space Sci Rev 214, 19 (2018). Eigenbrode, J. L. et al. Science, 360, 1096-1101 (2018). Gartman, A. et al. ACS ESC 1, 5, 277-287 (2017). Glavin, D. P. et al. Chem. Rev. 120, 11, 4660-4689 (2020). Goesmann, F. et al. Astrobiology, 17 (6-7), 655-685 (2017). Hand, K. Report of the Europa Lander science definition team, NASA (2017). Hoehler, T. M., Planetary Astrobiology (2020). Jia, X. et al. Nat. Astro. 2, 459-464 (2018). Johnson, S. S. et al. Astrobiology 18, 915-922 (2018). Kempes, C. P. et al. Front. Ecol. Evol. 7,242 (2019). Kim, H. et al. Sci. Adv. 5, eaau0149 (2019). Klaus, K. K. et al. AAS/Div. Planet. Sci. Abstracts 45, 211.14 (2013). Klein, H. P. Rev. Geophys. 17, 1655-1662 (1979). Levin, G. V., \& Straat P. A. Astrobiology 16, 798-810 (2016). Li, X. et al. Int. J. Mass Spec., 422, 177 - 187 (2017). Libby, E. et al., PLoS Comp Biol 15, 7 (2019). Mahaffy, P. R. et al. Space Sci. Rev. 170, $401-478$ (2012). Marshall, S. M. et al. Philos. Trans. R. Soc. Math. Phys. Eng. Sci. 375, 1-13 (2017). Nadeau, J. et al. Astrobiology 16, 755-774 (2016). NASA (2019). NASEM “An Astrobiology Strategy for the Search for Life in the Universe." (2018). Niemann, H. B. et al. L. Geophys. Res. Planets 115 (2010). Orosei, R. et al. Science 361, 490-493 (2018). Postberg, F. et al. Nature 558, 564-568 (2018). Rummel, J. D. Astrobiology 19, 624-627 (2019). Stoltenburg, R. et al. Biomol. Eng. 24, 381-403 (2007). Sun, H. \& Zu, Y. Molecules 20, 11959-11980 (2015). Steele, A. et al. Meteor. \& Planet. Sci. 51, 11, 2203-2225 (2016) Theiling, B. et al. NASEM, Planetary Science \& Astrobiology Decadal Survey White Paper (2020). Walker, S. I. Nature 569, 36 (2019). 